\title{
Using MicroCT to evaluate stain penetration and establish fiducial marks for high throughput electron microscopy.
}

JoAnn Buchanan ${ }^{1 *}$, Marc M. Takeno ${ }^{1}$, Agnes L. Bodor ${ }^{1}$, Daniel J. Bumbarger ${ }^{1}$, E.Froudarakis ${ }^{2}$, Jacob

Reimer ${ }^{2}$, Andres S. Tolias ${ }^{2}$, R. Clay Reid ${ }^{1}$, and Nuno M. da Costa ${ }^{1}$

${ }^{1}$ Allen Institute for Brain Science. Seattle, WA, USA.

${ }^{2}$ Baylor College of Medicine, Dept. of Neuroscience, Houston, TX, USA.

* Corresponding author: buchsmith@gmail.com

Mapping the wiring diagram of the neocortex constrains models of computation by the structural components of the brain. Electron microscopy (EM) remains the gold standard to identify these components since it has adequate resolution to identify thin wires (the axons) of micro connectivity and the synapses they form [1]. To map a full cortical column of cortex, large volumes $\left(1 \mathrm{~mm}^{3}\right)$ are needed in order to include the local connections of a mouse cortical microcircuit. Here, we describe an EM pipeline to scale up technology to collect petascale sized datasets containing a full local cortical microcircuit in mouse visual cortex. We supplement datasets with functional studies of neurons whose calcium activity has been imaged in vivo using 2 and 3 photon( $(\mathrm{P})$ imaging. Our goal was to prepare high contrast samples of 1.2 thick vibratome slices of previously $2 \mathrm{P}$ imaged visual cortex for serial section electron microscopy and correlate it with the functional data. To do this, we needed to cut $>25,000$ thin sections $40 \mathrm{~nm}$ in thickness for 3D reconstruction and automatic segmentation.

Preparation of $1 \mathrm{~mm}^{3}$ and larger specimens by EM presents technical challenges. The foremost problem is osmium tetroxide $\left(\mathrm{OsO}_{4}\right)$ penetration into the samples [2]. Osmium tetroxide $\left(\mathrm{OsO}_{4}\right)$ is paramount for successful EM sample preparation, since it acts as both a fixative and a stain, lending contrast to an otherwise transparent specimen. Osmium diffuses into biological samples slowly, on the order of $800 \mathrm{um}$ deep in the first hour and then forms a barrier to further penetration as time progresses. This leads to a gradation of staining, primarily in samples over $1 \mathrm{~mm}$ thick. In addition, the oxidation of the tissue by the osmium reaction causes it to become blackened and opaque, obscuring the anatomical details of the tissue. These anatomical details, such as cell soma location and blood vessel patterns, are needed to establish precise location of the region of interest (ROI) of the previously imaged cells in the resin blocks before trimming and cutting can begin. Therefore, we utilized MicroCT (micro computed tomography), a form of 3D x-ray imaging that can image the sample non-destructively and allow observation of fine cellular details and stain density [3].

Since we wanted to avoid post-staining of 25,000 sections, we utilized a specially designed protocol tor en bloc staining. This protocol utilized multiple rounds of Os staining combined with amplification with thiocarbohydrazide (OTO) to highlight membranes [4,5]. Increased time and addition of solvents was required to increase Os penetration. Applications of the heavy metals lead nitrate and uranyl acetate were added en bloc to further contrast the sample prior to dehydration and infiltration with resin. Mice P60114 were perfusion fixed, using a mixture of paraformaldehyde and glutaraldehyde. After perfusion the brain was removed, and the imaged area was marked with toluidine blue dye prior to vibratome cutting to ensure placement in the slice. This dye stained the surface of the brain and allowed visualization of the overlying blood vessel pattern. This permitted precision vibratome slicing, ensuring adequate margins around the ROI area. Following slicing, the samples were washed in 0.1 cacodylate buffer then transferred to vials for heavy metal processing and resin embedding. We sent resin blocks containing the ROI to 
Cornell for MicroCT imaging to evaluate stain penetration and overall suitability for cutting. Specifically, we looked for signs of cracks in the sample and inadequate stain penetration causing an unstained core in the tissue center. Resulting images helped chose the best samples for thin section and allowed exact overlay of LM data with the resin block. Finally, the blood vessel pattern was overlaid to map out exact position of the ROI in resin block. In total, we prepared 8 mice for analysis by TEM for quality of tissue fixation, stain penetration and block hardness. Final sample choice considered stain penetration, ultrastructure, contrast, ROI placement and physiological data. The best sample was registered with ROI fiducial marks and trimmed for collection of $25 \mathrm{~K}$ serial sections using an ATUM (automatic tape collection ultramicrotome).

\section{References:}

[1] EG Gray Nature 183 (1959), p1592.

[2] W Dempster, American Journal of Anatomy 107 (1960) p59.

[3] J Tapia et al., Nature Protocols 7 (2012), p193.

[4] Y Hua, P Laserstein and M Helmstaedter, Nature Communications 6 (2015) p1.

[5] E Scheller et al., Methods in enzymology 537 (2014) p123.

[6] Imaging data was acquired through the Cornell University Biotechnology Resource Center, with NIH 1S10OD012287 funding for the ZEISS-Xradia Versa 520 X-ray Microscope.

[7] Supported by the Intelligence Advanced Research Projects Activity (IARPA) via Department of Interior/ Interior Business Center (DoI/IBC) contract number D16PC00004. The U.S. Government is authorized to reproduce and distribute reprints for Governmental purposes notwithstanding any copyright annotation thereon. Disclaimer: The views and conclusions contained herein are those of the authors and should not be interpreted as necessarily representing the official policies or endorsements, either expressed or implied, of IARPA, DoI/IBC, or the U.S. Government
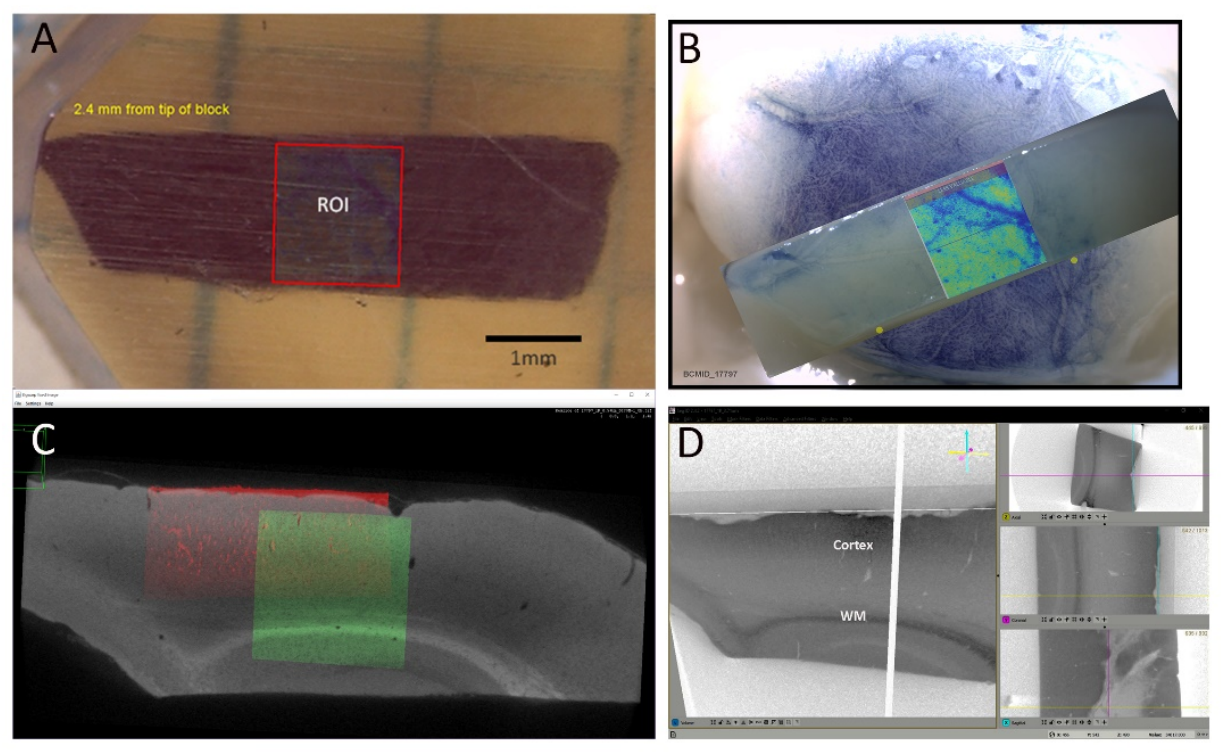

Figure 1.A) Embedded tissue sample in resin block. Region of interest (ROI) is labelled. Scale bar $1 \mathrm{~mm}$ B) Mouse brain stained with toluidine blue to reveal vasculature and the ROI overlay. C) MicroCT image with overlays. Green box is the ROI and red rectangle shows the 2P fluorescence signal overlay from the live imaging. D) Different views from MicroCT imaging shows even osmium staining throughout the sample. 